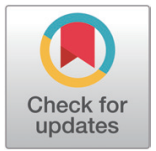

Received: Dec 3, 2020

Revised: Dec 14, 2020

Accepted: Dec 14, 2020

${ }^{*}$ Corresponding author

Minjung Yoon

Department of Animal Science and

Biotechnology, Kyungpook National

University, Sangju 37224, Korea.

Tel: +82-54-530-1233

E-mail: mjyoon@knu.ac.kr

Copyright $\odot 2021$ Korean Society of Animal Sciences and Technology.

This is an Open Access article distributed under the terms of the Creative Commons Attribution Non-Commercial License (http:// creativecommons.org/licenses/by$\mathrm{nc} / 4.0 /$ ) which permits unrestricted non-commercial use, distribution, and reproduction in any medium, provided the original work is properly cited.

ORCID

Junyoung Kim

https://orcid.org/0000-0003-0711-5479

Youngjae Park

https://orcid.org/0000-0003-0038-7615

Eun Joong Kim

https://orcid.org/0000-0002-5962-6994

Heejun Jung

https://orcid.org/0000-0001-9608-1412 Minjung Yoon

https://orcid.org/0000-0001-9112-1796

Competing interests

No potential conflict of interest relevant to this article was reported.

\section{Relationship between oxytocin and serotonin and the fearfulness, dominance, and trainability of horses}

\author{
Junyoung Kim ${ }^{1}$, Youngjae Park ${ }^{2}$, Eun Joong Kim ${ }^{1}$, Heejun $\mathrm{Jung}^{3}$ and \\ Minjung Yoon ${ }^{1 *}$
}

${ }^{1}$ Department of Animal Science and Biotechnology, Kyungpook National University, Sangju 37224, Korea

${ }^{2}$ Department of Equine Industry and Sports with Therapeutic Riding, Jeonju Kijeon College, Jeonju 54989 , Korea

${ }^{3}$ Department of Anatomy, School of Medicine, Kyungpook National University, Daegu 41944, Korea

\begin{abstract}
Oxytocin (OXT) and serotonin (5-HT) are essential neurotransmitters associated with the behavior of animals. Recently, we found that the plasma concentration of OXT is positively correlated with horse docility and friendliness toward humans. However, the relationships between the neurotransmitters and other temperaments such as fearfulness, dominance, and trainability are unknown. This study aimed to identify whether the plasma concentration of OXT or $5-\mathrm{HT}$ is correlated with fearfulness, dominance, and trainability of horses. Blood samples of 34 horses were collected at the Horse Industry Complex Center of Jeonju Kijeon College. The concentration of OXT and 5-HT was measured in the plasma samples using enzyme-linked immunosorbent assays. The fearfulness, dominance, and trainability of horses were scored by three professors who were very familiar with the horses. One-way analysis of variance with the least significant difference post-hoc analysis was used to compare the scores for fearfulness and dominance among groups. The trainability of horses was compared using the student $t$-test. The $5-\mathrm{HT}$ was negatively correlated with dominance, but it had no relation with fearfulness. The OXT appeared to be negatively correlated with fearfulness and dominance in horses. Furthermore, OXT was positively correlated with the trainability of horses. Additionally, 5-HT appeared to enhance trainability. In conclusion, the concentration of OXT or $5-\mathrm{HT}$ in horse blood plasma can be used as a biomarker to monitor the fearfulness, dominance, or trainability of horses.
\end{abstract}

Keywords: Horses, Oxytocin, Serotonin, Fearfulness, Dominance, Trainability

\section{INTRODUCTION}

The unpredictable behavior of horses can cause substantial problems [1]. Generally, horse behavior is related to the temperament of horses. Thus, the temperament of horses should be determined to prevent potential hazards during horse management $[2,3]$. Temperament is regarded as a collection of 
Funding sources

This research was supported by the Basic

Science Research Program through

the National Research Foundation of

Korea (NRF) funded by the Ministry of

Education, Science, and Technology

(2019R1A2C1003701).

Acknowledgements

The authors would like to thank Yeonju Choi, Youngwook Jung, Geumhee Lee (Kyungpook National University, the Korea), and Doyoung Jeong (Jeonju Kijeon College, the Korea) for their assistance with data collection. Also, the authors would like to thank Korean Racing Authority for their supports.

Availability of data and material Upon a reasonable request, the datasets of this study can be available from the corresponding author.

Authors' contributions

Conceptualization: Kim J.

Data curation: Kim EJ, Yoon M.

Formal analysis: Kim J, Yoon M.

Methodology: Kim J.

Software: Kim J.

Validation: Jung $\mathrm{H}$.

Investigation: Kim J, Park Y.

Writing - original draft: Kim J, Jung $\mathrm{H}$.

Writing - review \& editing: Kim EJ, Yoon M.

Ethics approval and consent to participate The research was approved by the Animal Experimentation Ethics Committee of Kyungpook National University (permit number: 2020-0131). behavioral trends based on the circumstances that animals experience from birth to maturity $[4,5]$. Thus, it is possible to predict the behavior of horses by assessing their temperament. Interestingly, there is strong evidence that the temperament of animals is associated with neuroendocrine systems. In rodents, oxytocin (OXT) and serotonin (5-hydroxytryptamine, 5-HT) are related to aggression, sociality, or affiliation [6,7]. A correlation between OXT concentrations and social behavior has also been found for humans $[8,9]$. Based on these results, the temperament of horses can be assessed by measuring blood concentrations of neurotransmitters. Recently, we reported that the concentration of OXT in horse plasma is positively correlated with docility and friendliness toward humans [10]. However, the correlation between the concentration of OXT or 5-HT in horse plasma with other characteristics of temperament, such as fearfulness, dominance, and trainability still needs to be elucidated.

In other species, OXT is released into the bloodstream by nerve signals, affecting animal behavior for a long time [11,12]. OXT decreases the fear of rats by directly affecting the amygdala [13], and it plays a role in inhibiting the ignition of noradrenergic locus coeruleus neurons, thereby restraining dominance and aggression in rats [14,15].

$5-\mathrm{HT}$ is one of the key factors determining the temperament of animals [16,17]. In pigs, tail biting is a prevalent problem caused by fearfulness and stress [18]. Tail biters have lower blood 5-HT concentrations than pigs that do not tail bite [19]. Additionally, higher concentrations of 5-HT in hamsters leads to lower levels of dominance behavior [20].

Based on findings for horses and other species, it is hypothesized that horses with a high concentration of OXT or 5-HT show lower levels of fearfulness and dominance behavior. Also, as sociality may also be associated with trainability, the OXT or 5-HT may be possibly associated with the trainability of horses. Thus, the objectives of this study were to identify whether the concentration of OXT or 5-HT in horse plasma is correlated with fearfulness, dominance, or trainability. The results of this study can be used to monitor temperament or specific behavioral tendencies and to inform suitable management and breeding programs of horses [21].

\section{MATERIALS AND METHODS}

\section{Animals}

This study was conducted at the Horse Industry Complex Center of Jeonju Kijeon College in Korea. The research was approved by the Animal Experimentation Ethics Committee of Kyungpook National University (permit number: 2020-0131). A total of 34 horses (aged 2-24 years; 23 mares and 11 geldings), comprised of 15 Thoroughbreds, nine Ponies, five Warmbloods, and five Haflingers, were used for this study. The horses were housed in individual stables $(3.5 \times 3.5$ $\mathrm{m})$ with an automatic water supply. Horses were fed with the total amount of roughage (1.5\% of body weight $[\mathrm{BW}])$ and concentrate $(0.5 \%$ of $\mathrm{BW})$ per day. Horses were used for several purposes such as competition for show-jumping or dressage, therapeutic riding, or schooling.

\section{Blood sampling}

Approximately $10 \mathrm{~mL}$ of blood samples were collected from the jugular vein of horses. Collected samples were immediately placed in an EDTA tube and stored in a $4^{\circ} \mathrm{C}$ icebox. To check the health status of the horses, the complete blood count test was immediately performed on-site using a VETSCAN HM5 Hematology Analyzer (ABAXIS, Union City, CA, USA). Blood samples of horses with no health problem and normal neutrophil/lymphocyte ratio were used. The blood samples were transported to the laboratory within $6 \mathrm{~h}$ and then, they were centrifuged to obtain plasma using a centrifuge machine (Labogene $1248 \mathrm{R}$, Gyrozen, Daejeon, Korea) at $25^{\circ} \mathrm{C}$ for 10 
mins at $1,500 \mathrm{~g}$. The plasma was collected and kept at $-20^{\circ} \mathrm{C}$ until further analysis.

\section{Enzyme-linked immunosorbent assay analysis}

The concentration of OXT and 5-HT in each plasma sample was analyzed using an enzymelinked immunosorbent assay (ELISA) kit (OXT: MBS033475, 5-HT: MBS037450, MyBioSource, San Diego, CA, USA). The sensitivities of 5-HT and OXT ELISA kit were $2.0 \mathrm{ng} / \mathrm{mL}$ and 1.0 $\mathrm{pg} / \mathrm{mL}$, respectively. The plasma samples were read at $450 \mathrm{~nm}$ on a Sunrise absorbance microplate reader (Tecan, Männedorf, Switzerland). The intra-assay variations for 5-HT and OXT were 3.55\% and 3.23\%, respectively. The inter-assay variations for 5-HT and OXT were $7.80 \%$ and $8.67 \%$, respectively.

\section{Questionary for the assessment of horse temperaments}

The degree of fearfulness, dominance, and trainability of horses were scored by three professors who were familiar with the horses. The score for each temperament of horses was given based on the criterion (Table 1). The degree of fearfulness was indicated by how much fear the horses displayed to unfamiliar reactions and how far they kept their distance from humans so that they were not caught [22]. The degree of dominance was indicated by the tendency of the horses to gain the upper hand over strangers or other horses and get priority of access to feed [23, 24]. The degree of trainability was indicated by the willingness of horses to be trained and the time required to achieve the desired response [25]. The scores for each temperament were set at zero to five points; low (zero or one point), medium (two or three), high (four or five). Each grade of horse temperament was adopted as the average value of the points.

\section{Statistical analysis}

All statistical analyses performed in this study used SPSS Statistics V25 (IBM, Armonk, NY, USA). One-way analysis of variance with the least significant difference post-hoc analysis was used to compare the concentration of neurotransmitters among three groups with different degrees of fearfulness and dominance. The concentration of neurotransmitters was compared between medium and high trainability groups using the student $\mathrm{t}$-test. The values whose IQRs exceed two terms of the first and third quartile of raw data were excluded as outliers. $p$-values below 0.05 were considered significant. All data were presented as mean \pm standard error (Tables 2,3, and 4).

\section{RESULTS}

\section{Relationship between plasma concentration of oxytocin and serotonin and the fearfulness of horses}

The plasma concentration of OXT seems to be negatively correlated with the degree of fearfulness. Horses with lower plasma OXT concentrations appear to show a higher degree of fearfulness although it was not statistically significant $(p=0.054$, Table 2$)$. However, no difference in plasma

Table 1. The criteria used for assessing equine temperament

\begin{tabular}{lllr}
\hline Temperament & \multicolumn{1}{c}{ Behavioral characteristics } & \multicolumn{2}{c}{ Score system } \\
\hline Fearfulness & $\begin{array}{l}\text { Keeping distance from someone unfamiliar } \\
\text { Reacting a rapid and excessive response to new stimuli }\end{array}$ & High & (4-5) \\
Dominance & $\begin{array}{l}\text { Trying to gain the upper hand over someone unfamiliar } \\
\text { Trying to gain the upper hand in the fight for food }\end{array}$ & Medium & $(2-3)$ \\
Trainability & $\begin{array}{l}\text { Willing to follow instruction during overall training sessions } \\
\text { Achieving the training goal sooner }\end{array}$ & Low & $(0-1)$ \\
\hline
\end{tabular}


5-HT concentrations was found among high, medium, and low fearfulness groups (Table 2).

\section{Relationship between plasma concentration of oxytocin and serotonin and the dominance of horses}

The plasma OXT concentrations were not significantly different among high, medium, and low dominance groups. However, horses with low plasma OXT concentrations tended to be more dominant than those with high plasma OXT concentrations ( $p=0.087$, Table 3$)$. The plasma 5-HT concentration in the high dominance group was significantly lower than those in the low and medium dominance groups ( $p=0.009$, Table 3$)$. Interestingly, there was no significant difference in the plasma 5-HT concentration between the medium and low dominance groups (Table 3).

\section{Relationship between plasma concentration of oxytocin and serotonin and the trainability of horses}

There was a significant positive correlation between the plasma OXT concentration and the degree of trainability (Table 4). The horses grouped in the high trainability had significantly higher plasma OXT concentrations compared with those grouped in the medium trainability $(p=0.02$, Table 4$)$. The plasma concentration of 5-HT was not significantly correlated with the degree of trainability. However, the plasma 5-HT concentration tended to be higher in the group of high trainability group compared with those in the medium trainability $(p=0.054$, Table 4$)$.

\section{DISCUSSION}

This study was performed to determine the relationship between plasma concentrations of OXT and 5-HT with fearfulness, dominance, and the trainability of horses. We found that plasma

Table 2. The mean concentrations of oxytocin and serotonin in horse blood plasma for different degrees of fearfulness in horses

\begin{tabular}{lccc}
\hline \multirow{2}{*}{ Neurotransmitters } & \multicolumn{3}{c}{ Degree of fearfulness } \\
\cline { 2 - 4 } & Low $(\mathbf{n}=\mathbf{1 3})$ & Medium $(\mathbf{n}=\mathbf{5})$ & High $(\mathbf{n}=\mathbf{1 1})$ \\
\hline OXT $(\mathrm{pg} / \mathrm{mL})$ & $47.33 \pm 2.42$ & $46.13 \pm 2.53$ & $41.16 \pm 1.92$ \\
$5-\mathrm{HT}(\mathrm{ng} / \mathrm{mL})$ & $94.54 \pm 5.99$ & $103.19 \pm 7.39$ & $82.85 \pm 6.06$ \\
\hline
\end{tabular}

Table 3. The mean concentrations of oxytocin and serotonin in horse blood plasma for different degrees of dominance in horses

\begin{tabular}{lccl}
\hline \multirow{2}{*}{ Neurotransmitters } & \multicolumn{3}{c}{ Degree of dominance } \\
\cline { 2 - 4 } & Low $(\mathbf{n}=\mathbf{9})$ & Medium $(\mathbf{n}=17)$ & High $(\mathbf{n}=\mathbf{3})$ \\
\hline OXT $(\mathrm{pg} / \mathrm{mL})$ & $47.86 \pm 2.78$ & $44.19 \pm 1.76$ & $38.89 \pm 3.94$ \\
$5-\mathrm{HT}(\mathrm{ng} / \mathrm{mL})$ & $98.81 \pm 6.95^{\mathrm{a}}$ & $93.54 \pm 3.97^{\mathrm{a}}$ & $58.96 \pm 12.08^{\mathrm{b}}$ \\
\hline
\end{tabular}

$, \mathrm{a}, \mathrm{b}$ Means in the same row with different letters are significantly different $(p<0.05)$.

Table 4. The mean concentrations of oxytocin and serotonin in horse blood plasma for different degrees of trainability of horses

\begin{tabular}{lcl}
\hline \multirow{2}{*}{ Neurotransmitters } & \multicolumn{2}{c}{ Degree of trainability } \\
\cline { 2 - 3 } & Medium $(\mathbf{n}=16)$ & High $(\mathbf{n}=13)$ \\
\hline OXT $(\mathrm{pg} / \mathrm{mL})$ & $41.82 \pm 1.56^{\mathrm{a}}$ & $48.42 \pm 2.25^{\mathrm{b}}$ \\
$5-\mathrm{HT}(\mathrm{ng} / \mathrm{mL})$ & $84.86 \pm 5.21$ & $99.89 \pm 5.20$ \\
\hline
\end{tabular}

, $\mathrm{a} b$ Means in the same row with different letters are significantly different $(p<0.05)$. 
OXT concentration and the degree of fearfulness appeared to be negatively correlated, which is in agreement with other studies showing that OXT reduces fearfulness in many species, including humans [26]. In humans, it was found that treatment with OXT reduced the fearfulness of individuals suffering from social anxiety disorder [26]. Furthermore, OXT has been shown to enhance trust and commitment to others in situations of possible social risks in interpersonal relationships by reducing fearfulness [27]. A group of rats expressing a low concentration of OXT receptor in the central nucleus of the amygdala showed higher fearfulness and vice versa [28]. These results are consistent with our finding that horses with a low concentration of plasma OXT tended to be fearful. In contrast to OXT, the plasma concentration of 5-HT was not correlated with the degree of fearfulness in horses. The absence of a relationship between 5-HT concentration and the degree of fearfulness was also found in pigs. Pigs raised in a barren cage and assumed more fearful showed no significant difference in blood concentrations of 5-HT when compared with those raised in enriched cages and assumed less fearful [19]. This result indicates that 5-HT is not associated with the fearful temperament of horses.

In this study, a negative correlation was found between the plasma concentration of 5-HT and horse dominance. Similar results were reported for dominant rats. Dominant rats treated with fluoxetine, a selective 5-HT reuptake inhibitor, showed a more equal partnership with subordinate rats [29], proving that 5-HT inhibits dominance in rats. This result supports the fact horses with higher concentrations of 5-HT in the plasma have a less dominant temperament.

There was a negative correlation between the concentration of plasma OXT and the level of dominance in horses, which is similar to findings that OXT leads to better interpersonal relationships in macaque monkeys by flattening the dominance hierarchy [30]. Animals with a dominant temperament show fewer interactions with other animals and humans [31-33]. A group of animals with fewer interactions with the same species or humans had a low concentration of OXT. A group of sows showing low concentrations of OXT had fewer interactions with humans [34]. In cattle, heifers with low postnatal plasma OXT concentrations were found to show less affinity behavior than those with higher postnatal plasma OXT concentrations [35]. Overall, OXT plays a role in down-regulating dominance, which is followed by more social behaviors. Thus, previous research supports our finding that OXT lowers the dominant temperament in horses.

Data in this study indicated that the plasma OXT concentration was positively correlated with trainability in horses. The same result was also demonstrated in dogs. Due to the influence of OXT, dogs showing more willingness to work with trainers exhibited high levels of trainability and getting trained quicker [36]. This result supports our speculation that a high concentration of OXT can enhance the interrelationship between horses and humans, and this relationship is associated with higher trainability.

In this study, although it was not statistically significant, a positive correlation between the concentration of 5-HT in horse blood plasma and the trainability of horses appeared to be present. Interestingly, in humans, the depletion of 5-HT caused several problems such as decreasing the level of cooperation and being discredited between partners [37]. Combining the results in horses and humans, 5-HT appears to play a positive role in cooperation and trust, leading to high trainability.

\section{CONCLUSION}

The OXT and 5-HT may be used as biomarkers to monitor the fearfulness, dominance, and trainability of horses. As a further study, the effects of treatment with these neurotransmitters on transit temperaments of horses should be conducted. 


\section{REFERENCES}

1. Watt GM, Finch CF. Preventing equestrian injuries. Sports Med. 1996;22:187-97. https://doi. org/10.2165/00007256-199622030-00005

2. Guyton K, Houchen-Wise E, Peck E, Mayberry J. Equestrian injury is costly, disabling, and frequently preventable: the imperative for improved safety awareness. Am Surg. 2013;79:76-83. https://doi.org/10.1177/000313481307900134

3. Thompson K, McGreevy P, McManus P. A critical review of horse-related risk: a research agenda for safer mounts, riders and equestrian cultures. Animals. 2015;5:561-75. https://doi. org/10.3390/ani5030372

4. Bates JE. Temperament in infancy. In Osofsky JD, editor. Handbook of infant development. New York, NY: John Wiley \& Sons; 1987.

5. Buss AH, Chess S, Goldsmith HH, Hinde RA, McCall R, Plomin R, et al. What is temperament: four approaches. Child Dev. 1987;58:505-29. https://doi.org/10.2307/1130527

6. Koolhaas J, De Boer SF, Coppens CM, Buwalda B. Neuroendocrinology of coping styles: towards understanding the biology of individual variation. Front Neuroendocrinol. 2010;31:30721. https://doi.org/10.1016/j.yfrne.2010.04.001

7. Giacalone E, Tansella M, Valzelli L, Garattini S. Brain serotonin metabolism in isolated aggressive mice. Biochem Pharmacol. 1968;17:1315-27. https://doi.org/10.1016/00062952(68)90069-5

8. Churchland PS, Winkielman P. Modulating social behavior with oxytocin: how does it work? What does it mean? Horm Behav. 2012;61:392-9. https://doi.org/10.1016/j.yhbeh.2011.12.003

9. Kumsta R, Heinrichs M. Oxytocin, stress and social behavior: neurogenetics of the human oxytocin system. Curr Opin Neurobiol. 2013;23:11-6. https://doi.org/10.1016/j.conb.2012.09.004

10. Lee G, Yoon M. Association of plasma concentrations of oxytocin, vasopressin and serotonin with docility and friendliness of horses. Domest Anim Endocrinol. 2021;74:106482. https:// doi.org/10.1016/j.domaniend.2020.106482

11. Sabatier N, Caquineau C, Dayanithi G, Bull P, Douglas AJ, Guan XMM, et al. $\alpha$-Melanocyte-stimulating hormone stimulates oxytocin release from the dendrites of hypothalamic neurons while inhibiting oxytocin release from their terminals in the neurohypophysis. J Neurosci. 2003;23:10351-8. https://doi.org/10.1523/jneurosci.23-32-10351.2003

12. Ludwig M, Leng G. Dendritic peptide release and peptide-dependent behaviours. Nat Rev Neurosci. 2006;7:126-36. https://doi.org/10.1038/nrn1845

13. Francis DD, Champagne FC, Meaney MJ. Variations in maternal behaviour are associated with differences in oxytocin receptor levels in the rat. J Neuroendocrinol. 2000;12:1145-8. https://doi.org/10.1046/j.1365-2826.2000.00599.x

14. Uvnäs-Moberg K. Oxytocin may mediate the benefits of positive social interaction and emotions1. Psychoneuroendocrinology. 1998;23:819-35. https://doi.org/10.1016/s03064530(98)00056-0

15. Petersson M, Uvnäs-Moberg K, Erhardt S, Engberg G. Oxytocin increases locus coeruleus alpha 2-adrenoreceptor responsiveness in rats. Neurosci Lett. 1998;255:115-8. https://doi. org/10.1016/s0304-3940(98)00729-0

16. Jacobs BL, Azmitia EC. Structure and function of the brain serotonin system. Physiol Rev. 1992;72:165-229. https://doi.org/10.1152/physrev.1992.72.1.165

17. Bari A, Robbins TW. Inhibition and impulsivity: behavioral and neural basis of response control. Prog Neurobiol. 2013;108:44-79. https://doi.org/10.1016/j.pneurobio.2013.06.005

18. Taylor NR, Main DCJ, Mendl M, Edwards SA. Tail-biting: a new perspective. Vet J. 
2010;186:137-47. https://doi.org/10.1016/j.tvjl.2009.08.028

19. Ursinus WW, Van Reenen CG, Reimert I, Bolhuis JE. Tail biting in pigs: blood serotonin and fearfulness as pieces of the puzzle? PLOS ONE. 2014;9:e107040. https://doi.org/10.1371/ journal.pone. 0107040

20. Ferris CF, Delville Y. Vasopressin and serotonin interactions in the control of agonistic behavior. Psychoneuroendocrinology. 1994;19:593-601. https://doi.org/10.1016/0306-4530(94)90043-4

21. Mills D. Personality and individual differences in the horse, their significance, use and measurement. Equine Vet J.1998;30:10-3. https://doi.org/10.1111/j.2042-3306.1998.tb05137.x

22. McCall CA, Hall S, McElhenney WH, Cummins KA. Evaluation and comparison of four methods of ranking horses based on reactivity. Appl Anim Behav Sci. 2006;96:115-27. https:// doi.org/10.1016/j.applanim.2005.04.021

23. Houpt KA, Law K, Martinisi V. Dominance hierarchies in domestic horses. Appl Anim Ethol. 1978;4:273-83. https://doi.org/10.1016/0304-3762(78)90117-7

24. Weeks JW, Crowell-Davis SL, Caudle AB, Heusner GL. Aggression and social spacing in light horse (Equus caballus) mares and foals. Appl Anim Behav Sci. 2000;68:319-37. https:// doi.org/10.1016/s0168-1591(99)00126-4

25. Duberstein KJ, Gilkeson JA. Determination of sex differences in personality and trainability of yearling horses utilizing a handler questionnaire. Appl Anim Behav Sci. 2010;128:57-63. https://doi.org/10.1016/j.applanim.2010.09.012

26. Gorka SM, Fitzgerald DA, Labuschagne I, Hosanagar A, Wood AG, Nathan PJ, et al. Oxytocin modulation of amygdala functional connectivity to fearful faces in generalized social anxiety disorder. Neuropsychopharmacology. 2015;40:278-86. https://doi.org/10.1038/npp.2014.168

27. Kosfeld M, Heinrichs M, Zak PJ, Fischbacher U, Fehr E. Oxytocin increases trust in humans. Nature. 2005;435:673-6. https://doi.org/10.1038/nature03701

28. Francis DD, Champagne FC, Meaney MJ. Variations in maternal behaviour are associated with differences in oxytocin receptor levels in the rat. J Neuroendocrinol. 2000;12:1145-8. https://doi.org/10.1046/j.1365-2826.2000.00599.x

29. Knutson B, Panksepp J, Pruitt D. Effects of fluoxetine on play dominance in juvenile rats. Aggress Behav. 1996;22:297-307. https://doi.org/10.1002/(sici)1098-2337(1996)22:4<297::aidab5 $>3.0 . c 0 ; 2-\mathrm{i}$

30. Jiang Y, Platt ML. Oxytocin and vasopressin flatten dominance hierarchy and enhance behavioral synchrony in part via anterior cingulate cortex. Sci Rep. 2018;8:1-14. https://doi. org/10.1038/s41598-018-25607-1

31. Sapolsky RM. Stress in the wild. Sci Am. 1990;262:116-23. https://www.jstor.org/stable $/ 24996650$

32. Kotrschal K, Schöberl I, Bauer B, Thibeaut AM, Wedl M. Dyadic relationships and operational performance of male and female owners and their male dogs. Behav Process. 2009;81:383-91. https://doi.org/10.1016/j.beproc.2009.04.001

33. Tomasello M. The ultra-social animal. Eur J Soc Psychol. 2014;44:187-94. https://doi. org/10.1002/ejsp.2015

34. Rault JL. Effects of positive and negative human contacts and intranasal oxytocin on cerebrospinal fluid oxytocin. Psychoneuroendocrinology. 2016;69:60-6. https://doi.org/10.1016/j.psyneuen.2016.03.015

35. Yayou KI, Ito S, Yamamoto N. Relationships between postnatal plasma oxytocin concentrations and social behaviors in cattle. Anim Sci J.2015;86:806-13. https://doi.org/10.1111/asj.12363

36. Konno A, Inoue-Murayama M, Yabuta S, Tonoike A, Nagasawa M, Mogi K, et al. Effect of canine oxytocin receptor gene polymorphism on the successful training of drug detection dogs. 
J Hered. 2018;109:566-72. https://doi.org/10.1093/jhered/esy012

37. Wood RM, Rilling JK, Sanfey AG, Bhagwagar Z, Rogers RD. Effects of tryptophan depletion on the performance of an iterated Prisoner's Dilemma game in healthy adults. Neuropsychopharmacology. 2006;31:1075-84. https://doi.org/10.1038/sj.npp.1300932 